\title{
Detecting the Earliest Galaxies through Two New Sources of 21 Centimeter Fluctuations
}

\section{Citation}

Barkana, Rennan, and Abraham Loeb. 2005. "Detecting the Earliest Galaxies through Two New Sources of 21 Centimeter Fluctuations." The Astrophysical Journal 626 (1): 1-11. https:// doi.org/10.1086/429954.

\section{Permanent link}

http://nrs.harvard.edu/urn-3:HUL.InstRepos:41393433

\section{Terms of Use}

This article was downloaded from Harvard University's DASH repository, and is made available under the terms and conditions applicable to Other Posted Material, as set forth at http:// nrs.harvard.edu/urn-3:HUL.InstRepos:dash.current.terms-of-use\#LAA

\section{Share Your Story}

The Harvard community has made this article openly available.

Please share how this access benefits you. Submit a story.

Accessibility 


\title{
DETECTING THE EARLIEST GALAXIES THROUGH TWO NEW SOURCES OF 21 CENTIMETER FLUCTUATIONS
}

\author{
Rennan Barkana ${ }^{1}$ AND Abraham Loeb ${ }^{2}$ \\ Received 2004 October 5; accepted 2005 February 25
}

\begin{abstract}
The first galaxies that formed at a redshift $z \sim 20-30$ emitted continuum photons with energies between the Ly $\alpha$ and Lyman limit wavelengths of hydrogen, to which the neutral universe was transparent except at the Lyman series resonances. As these photons redshifted or scattered into the $\mathrm{Ly} \alpha$ resonance, they coupled the spin temperature of the $21 \mathrm{~cm}$ transition of hydrogen to the gas temperature, allowing it to deviate from the microwave background temperature. We show that the fluctuations in the radiation emitted by the first galaxies produced strong fluctuations in the $21 \mathrm{~cm}$ flux before the Ly $\alpha$ coupling became saturated. The fluctuations were caused by biased inhomogeneities in the density of galaxies, along with Poisson fluctuations in the number of galaxies. Observing the power spectra of these two sources would probe the number density of the earliest galaxies and the typical mass of their host dark matter halos. The enhanced amplitude of the $21 \mathrm{~cm}$ fluctuations from the era of Ly $\alpha$ coupling improves considerably the practical prospects for their detection.
\end{abstract}

Subject headings: cosmology: theory — galaxies: formation — galaxies: high-redshift

Online material: color figures

\section{INTRODUCTION}

The reionization history of cosmic hydrogen, left over from the big bang, provides crucial fossil evidence for when the first stars and black holes formed in the infant universe (Barkana \& Loeb 2001). The hyperfine spin-flip transition of neutral hydrogen ( $\mathrm{H} \mathrm{I})$ at a wavelength of $21 \mathrm{~cm}$ is potentially the most promising probe of the cosmic gas before reionization ended. Observations of this line at a wavelength of $21(1+z) \mathrm{cm}$ can be used to slice the universe as a function of redshift $z$ and obtain a three-dimensional map of the diffuse $\mathrm{H}$ I distribution in it (Hogan \& Rees 1979). The $21 \mathrm{~cm}$ signal vanished at redshifts $z \gtrsim 200$, when the small residual fraction of free electrons after cosmological recombination kept the gas kinetic temperature, $T_{k}$, close to the temperature of the cosmic microwave background (CMB), $T_{\gamma}$. Subsequently, between $200 \gtrsim z \gtrsim 30$ the gas cooled adiabatically, faster than the CMB, and atomic collisions kept the spin temperature $T_{s}$ of the hyperfine level population below $T_{\gamma}$, so that the gas appeared in absorption (Scott \& Rees 1990). Primordial density inhomogeneities imprinted a three-dimensional power spectrum of $21 \mathrm{~cm}$ flux fluctuations on scales down to $\$ 10$ comoving kpc, making it the richest data set on the sky (Loeb \& Zaldarriaga 2004). As the Hubble expansion continued to rarefy the gas, radiative coupling of $T_{S}$ to $T_{\gamma}$ dominated over collisional coupling of $T_{s}$ to $T_{k}$ and the $21 \mathrm{~cm}$ signal began to diminish.

However, as soon as the first galaxies appeared, the UV photons they produced between the Ly $\alpha$ and Lyman limit wavelengths propagated freely through the universe, redshifted or scattered into the Ly $\alpha$ resonance, and coupled $T_{s}$ and $T_{k}$ once again through the Wouthuysen-Field (Wouthuysen 1952; Field 1958) effect by which the two hyperfine states are mixed through the absorption and reemission of an Ly $\alpha$ photon (Madau et al. 1997; Ciardi \& Madau 2003). Emission of UV photons above

\footnotetext{
1 School of Physics and Astronomy, The Raymond and Beverly Sackler Faculty of Exact Sciences, Tel Aviv University, Tel Aviv 69978, Israel; barkana@ wise.tau.ac.il.

2 Astronomy Department, Harvard University, 60 Garden Street, Cambridge, MA 02138; aloeb@cfa.harvard.edu.
}

the Lyman limit by the same galaxies initiated the process of reionization by creating ionized bubbles in the neutral gas around these galaxies. While UV photons were blocked at the neutral boundary of the bubbles, X-ray photons propagated farther into the bulk of the intergalactic gas and heated $T_{k}$ above $T_{\gamma}$ throughout the universe. Once $T_{s}$ grew larger than $T_{\gamma}$, the gas appeared in $21 \mathrm{~cm}$ emission with a brightness temperature that was asymptotically independent of $T_{s}$ and $T_{k}$. The ionized bubbles imprinted a knee in the power spectrum of $21 \mathrm{~cm}$ fluctuations (Zaldarriaga et al. 2004), which traced the topology of $\mathrm{H}$ I as the ionized bubbles percolated and completed the process of reionization (Furlanetto et al. 2004a).

Since the first galaxies represented rare peaks in the cosmic density field, their spatial distribution possessed unusually large fluctuations on large scales (Barkana \& Loeb 2004). In this paper we show that the resulting large-scale bias of the first galaxies produced substantial $21 \mathrm{~cm}$ fluctuations during the intermediate epoch when the first Ly $\alpha$ photons coupled $T_{s}$ to $T_{k}$. Since a relatively small number of galaxies contributed to the flux seen at any given point, Poisson fluctuations were significant as well, and we show that they produced correlated $21 \mathrm{~cm}$ fluctuations. Owing to the rapid evolution in the density of galaxies with cosmic time, there is a sharp decline in the abundance of sources with distance (corresponding to retarded cosmic time for the source emission due to the light-travel time) around each point in the intergalactic medium (IGM). Ultimately, there is an effective horizon around each point out to which sources may contribute to $21 \mathrm{~cm}$ fluctuations. In addition, the atomic physics of resonant scattering by hydrogen fixes different horizon distances for photons emitted in various wavelength intervals between Ly $\alpha$ and the Lyman limit; these horizons are set by the requirement that a given photon must redshift or scatter into the Ly $\alpha$ resonance at the point of interest. We incorporate all of these ingredients into our calculation.

The outline of the paper is as follows. In $\S 2$ we summarize the basic equations that describe the evolution of the spin temperature. In $\S 3$ we review the angular anisotropy of the $21 \mathrm{~cm}$ power spectrum due to peculiar velocities, as derived in our previous paper (Barkana \& Loeb 2005). We then calculate the flux 
of Ly $\alpha$ photons produced by the earliest galaxies ( $\S 4)$ and its fluctuations $(\S 5)$. Finally, we demonstrate how the resulting power spectrum of fluctuations in the $21 \mathrm{~cm}$ flux $(\S 6)$ can be used to study the properties of the earliest galaxies $(\S 7)$ and briefly summarize our main results $(\S 8)$. Throughout the paper we assume the concordance set of cosmological parameters (Spergel et al. 2003).

\section{SPIN TEMPERATURE HISTORY}

The $\mathrm{H}_{\text {I }}$ spin temperature, $T_{s}$, is defined through the ratio between the number densities of hydrogen atoms in the excited and ground-state levels, $n_{1} / n_{0}=\left(g_{1} / g_{0}\right) \exp \left(-T_{*} / T_{s}\right)$, where $\left(g_{1} / g_{0}\right)=3$ is the ratio of the spin degeneracy factors of the levels and $T_{*}=0.0682 \mathrm{~K}$. When the spin temperature is smaller than the CMB temperature, neutral hydrogen atoms absorb CMB photons. The resonant $21 \mathrm{~cm}$ absorption reduces the brightness temperature of the CMB by (Scott \& Rees 1990; Madau et al. 1997)

$$
T_{b}=\tau\left(\frac{T_{s}-T_{\gamma}}{1+z}\right)
$$

where the optical depth for resonant $\lambda=21 \mathrm{~cm}$ absorption is

$$
\tau=\frac{3 c \lambda^{2} h A_{10} n_{\mathrm{H}}}{32 \pi k_{\mathrm{B}} T_{s}(1+z)\left(d v_{r} / d r\right)},
$$

where $n_{\mathrm{H}}$ is the number density of hydrogen, $A_{10}=2.85 \times$ $10^{-15} \mathrm{~s}^{-1}$ is the spontaneous emission coefficient, and $d v_{r} / d r$ is the gradient of the radial velocity along the line of sight, with $v_{r}$ being the physical radial velocity and $r$ the comoving distance; on average $d v_{r} / d r=H(z) /(1+z)$, where $H$ is the Hubble parameter. The velocity gradient term arises since the $21 \mathrm{~cm}$ scattering cross section has a fixed thermal width that translates through the redshift factor $\left(1+v_{r} / c\right)$ to a fixed interval in velocity (Sobolev 1960). In this paper we consider the epoch long before complete reionization, and we assume that the hydrogen gas is almost entirely neutral.

The mean brightness temperature offset on the sky at redshift $z$ is

$$
T_{b}=28 \mathrm{mK}\left(\frac{\Omega_{b} h}{0.033}\right)\left(\frac{\Omega_{m}}{0.27}\right)^{-1 / 2}\left(\frac{1+z}{10}\right)^{1 / 2}\left(\frac{T_{s}-T_{\gamma}}{T_{s}}\right),
$$

where we have substituted the concordance values for the cosmological parameters $\Omega_{b}, h$, and $\Omega_{m}$. The spin temperature itself is coupled to $T_{k}$ through the spin-flip transition, which can be excited by atomic collisions or by the absorption of Ly $\alpha$ photons. Assuming that the photons near Ly $\alpha$ are redistributed in frequency according to the thermal velocities of the atoms (i.e., the spectrum is thermalized according to $T_{k}$ ), the combination that appears in $T_{b}$ becomes (Field 1958)

$$
\frac{T_{s}-T_{\gamma}}{T_{s}}=\frac{x_{\mathrm{tot}}}{1+x_{\mathrm{tot}}}\left(1-\frac{T_{\gamma}}{T_{k}}\right)
$$

where $x_{\text {tot }}=x_{\alpha}+x_{c}$ is the sum of the radiative and collisional threshold parameters. These parameters are

$$
x_{\alpha}=\frac{4 P_{\alpha} T_{*}}{27 A_{10} T_{\gamma}}
$$

and

$$
x_{c}=\frac{4 \kappa_{1-0}\left(T_{k}\right) n_{\mathrm{H}} T_{*}}{3 A_{10} T_{\gamma}}
$$

where $P_{\alpha}$ is the Ly $\alpha$ scattering rate that is proportional to the $\operatorname{Ly} \alpha$ intensity and $\kappa_{1-0}\left(T_{k}\right)$ is tabulated as a function of $T_{k}$ (Allison \& Dalgarno 1969; Zygelman 2005). Note that we have adopted a modified notation compared to the $y_{\alpha}$ and $y_{c}$ previously used (Field 1958; Madau et al. 1997), defining $x_{\alpha} \equiv y_{\alpha}\left(T_{\alpha} / T_{\gamma}\right)$ and $x_{c} \equiv$ $y_{c}\left(T_{k} / T_{\gamma}\right)$. The coupling of the spin temperature to the gas temperature becomes substantial when $x_{\text {tot }} \gtrsim 1$; in particular, $x_{\alpha}=$ 1 defines the thermalization rate (Madau et al. 1997) of $P_{\alpha}$, which is $7.69 \times 10^{-12}(1+z) / 10$ photons $\mathrm{s}^{-1}$.

\section{THE SEPARATION OF POWERS}

Although the mean $21 \mathrm{~cm}$ emission or absorption is difficult to measure owing to bright foregrounds, the unique character of the fluctuations in $T_{b}$ allows for a much easier extraction of the signal (Gnedin \& Shaver 2004; Zaldarriaga et al. 2004; Morales \& Hewitt 2004; Morales 2005; Santos et al. 2004). During the era of initial Ly $\alpha$ coupling, fluctuations in $T_{b}$ are sourced by fluctuations in the density $(\delta)$, temperature $\left(\delta_{T_{k}}\right)$, and radial velocity gradient of the gas, as well as the Ly $\alpha$ flux through $x_{\alpha}\left(\delta_{x_{\alpha}}\right)$. The fluctuations in the neutral fraction of the gas become important only later, when the volume filling fraction of ionized bubbles is substantial.

The above sources of fluctuations are all isotropic except for the velocity gradient term. Since this term involves radial projections, it inserts an anisotropy into the power spectrum (Kaiser 1987; Tozzi et al. 2000; Bharadwaj \& Ali 2004). The total power spectrum can be written as (Barkana \& Loeb 2005)

$$
\begin{aligned}
P_{T_{b}}(\boldsymbol{k})= & \mu^{4} P_{\delta}(k)+2 \mu^{2}\left[\beta P_{\delta}(k)+\frac{x_{\alpha}}{\tilde{x}_{\mathrm{tot}}} P_{\delta-\alpha}(k)\right] \\
& +\left[\beta^{2} P_{\delta}(k)+\left(\frac{x_{\alpha}}{\tilde{x}_{\mathrm{tot}}}\right)^{2} P_{\alpha}(k)+\frac{2 \beta x_{\alpha}}{\tilde{x}_{\mathrm{tot}}} P_{\delta-\alpha}(k)\right],
\end{aligned}
$$

where $\tilde{x}_{\text {tot }} \equiv\left(1+x_{\text {tot }}\right) x_{\text {tot }}, P_{\delta}$ and $P_{\alpha}$ are the power spectra of the fluctuations in density and in $x_{\alpha}$, respectively, and the power spectrum $P_{\delta-\alpha}$ is the Fourier transform of the cross-correlation function,

$$
\xi_{\delta-\alpha}(r)=\left\langle\delta\left(\boldsymbol{r}_{1}\right) \delta_{x_{\alpha}}\left(\boldsymbol{r}_{1}+\boldsymbol{r}\right)\right\rangle
$$

Here $\beta$ is given by (Barkana \& Loeb 2005)

$\beta=1+\frac{x_{c}}{\tilde{x}_{\mathrm{tot}}}+\left(\gamma_{a}-1\right)\left[\frac{T_{\gamma}}{T_{k}-T_{\gamma}}+\frac{x_{c}}{\tilde{x}_{\mathrm{tot}}} \frac{d \log \left(\kappa_{1-0}\right)}{d \log \left(T_{k}\right)}\right]$,

where the adiabatic index is $\gamma_{a}=1+\left(\delta_{T_{k}} / \delta\right)$. Also, $\mu$ is the cosine of the angle between the wavenumber $\boldsymbol{k}$ of the Fourier mode and the line-of-sight direction. Considering equation (7) as a polynomial in $\mu$, i.e.,

$$
P_{T_{b}}(\boldsymbol{k})=\mu^{4} P_{\mu^{4}}+\mu^{2} P_{\mu^{2}}+P_{\mu^{0}},
$$

we note that the power at just three values of $\mu$ is required in order to separate out observationally the coefficients of $1, \mu^{2}$, and $\mu^{4}$ for each $k$. In particular, we can construct a combination that 
probes whether some sources of flux fluctuations are uncorrelated with $\delta$, through (Barkana \& Loeb 2005)

$$
P_{\mathrm{un}-\delta}(k) \equiv P_{\mu^{0}}-\frac{P_{\mu^{2}}^{2}}{4 P_{\mu^{4}}}=\left(\frac{x_{\alpha}}{\tilde{x}_{\mathrm{tot}}}\right)^{2}\left(P_{\alpha}-\frac{P_{\delta}^{2}-\alpha}{P_{\delta}}\right) .
$$

Note that since $21 \mathrm{~cm}$ measurements on large angular scales cannot yield significant information (Barkana \& Loeb 2005), we focus in this paper on three-dimensional power spectra, which are directly measurable from $21 \mathrm{~cm}$ observations in small angular fields.

We note that several additional redshift effects do not significantly modify the $21 \mathrm{~cm}$ brightness fluctuations. First, consider an observed correlation function (or the equivalent power spectrum) that is calculated by averaging the product of the $21 \mathrm{~cm}$ temperatures at pairs of points within a small three-dimensional volume. We assume that this volume is centered on redshift $z$ and has a redshift width $\Delta z$ that corresponds to the observed frequency range used to calculate the correlation function at $z$. The change in the cosmic mean temperature $T_{b}\left(z^{\prime}\right)$ with redshift $z^{\prime}$ within this slice would produce variations in temperature even in a homogeneous expanding universe. However, these variations are coherent on the sky and have a very simple form. Denoting by $l$ the line-of-sight component of $\boldsymbol{r}$, where $l=0$ marks the center of the redshift slice and $\Delta l$ its width, the correlation function $\xi_{T_{b}}(r) \equiv$ $\left\langle\delta_{T_{b}}\left(\boldsymbol{r}_{1}\right) \delta_{T_{b}}\left(\boldsymbol{r}_{1}+\boldsymbol{r}\right)\right\rangle$ is modified to $\left\langle\left(\delta_{T_{b}}\left(\boldsymbol{r}_{1}\right)+l_{1} d_{l} T_{b}\right)\left[\delta_{T_{b}}\left(\boldsymbol{r}_{1}+\right.\right.\right.$ $\left.\left.\boldsymbol{r})+\left(l_{1}+l\right) d_{l} T_{b}\right]\right\rangle$, where we denote the derivative $\left[d T_{b}(z) / d l\right]$ at $z$ by $d_{l} T_{b}$. Noting that $\left\langle\delta_{T_{b}}\left(\boldsymbol{r}_{1}\right)\right\rangle$ and $\left\langle l_{1}\right\rangle$ are both zero within the slice, we find that $\xi_{T_{b}}(r)$ receives an extra term, $\left(d_{l} T_{b}\right)^{2}\left\langle l_{1}^{2}\right\rangle$. Since $\left\langle l_{1}^{2}\right\rangle=(\Delta l)^{2} / 12$, the finite redshift width $\Delta z$ adds to the power spectrum $P_{T_{b}}(\boldsymbol{k})$ a term

$$
P_{T_{b}: \Delta z}(\boldsymbol{k})=\frac{2}{3} \pi^{3}\left[\Delta z \frac{d T_{b}(z)}{d z}\right]^{2} \delta_{\mathrm{D}}^{3}(\boldsymbol{k})
$$

Thus, this effect does not modify the power spectrum at $k \neq 0$.

In addition, peculiar velocities change the apparent distance along the line of sight. Over a distance $l$, the unperturbed velocity $v=a H l$ receives a fractional perturbation $\delta_{v}(l)$, which is of order the density perturbation $\delta$ averaged on the scale $l$ (e.g., $\delta_{v}=-\delta / 3$ for a spherical top-hat perturbation out to radius $l$ ). The mean temperature $T_{b}$ is modified owing to this displacement by $\sim l \delta_{v}(l) d_{l} T_{b}$, which is smaller than $\delta$ by $\sim a l H / c$, which at $z=20$ is $l /(2 \mathrm{Gpc}) \ll 1$. Also, to first order we can neglect the effect of the peculiar velocities on the perturbation $\delta_{T_{b}}$ itself. Finally, gravitational potential fluctuations are $\sim 10^{-5}$ on the scale of the horizon and are suppressed relative to the density fluctuations by a factor of $\sim(\text { alH/c })^{2}$ on small scales. Note that $10^{-5}$ fluctuations are important in CMB observations, since at $z \sim 1100$ density fluctuations on small scales had not yet grown owing to the radiation pressure of the photon-baryon fluid; in the redshift range that we consider for $21 \mathrm{~cm}$ observations, however, density fluctuations are already at a level of $\sim 1 \%$ on $100 \mathrm{Mpc}$ scales (at $z=20$ ), and it would be very difficult to measure the $\sim 10^{-4}$ fluctuations on gigaparsec scales (Barkana \& Loeb 2005).

\section{THE Ly $\alpha$ FLUX OF GALAXIES}

The intensity of Ly $\alpha$ photons observed at a given redshift is due to photons that were originally emitted between the restframe wavelengths of $\operatorname{Ly} \alpha$ and the Lyman limit. Photons that were emitted below $\mathrm{Ly} \beta$ by some source simply redshift until they are seen by an atom at $z$ at the Ly $\alpha$ wavelength. Such photons can only be seen out to a distance corresponding to the redshift $z_{\max }(2)$, where $1+z_{\max }(2)=(32 / 27)(1+z)$, where $32 / 27$ is the ratio of $\operatorname{Ly} \alpha$ to $\operatorname{Ly} \beta$ wavelengths. Photons above the $\operatorname{Ly} \beta$ energy redshift until they reach the nearest atomic level $n$. The neutral IGM is opaque even to the higher levels and so the photon is absorbed and reemitted. During these scatterings, the photon is almost immediately downgraded to an $\operatorname{Ly} \alpha$ photon and then keeps on scattering, and so most of the atoms that interact with it encounter it as an Ly $\alpha$ photon. To be seen at the Ly $\alpha$ resonance at $z$, the photon must have been emitted below a redshift given by

$$
1+z_{\max }(n)=(1+z) \frac{\left[1-(n+1)^{-2}\right]}{\left(1-n^{-2}\right)} .
$$

Thus, we simply add up the number flux of photons emitted between consecutive atomic levels and integrate over sources out to the distance corresponding to the appropriate redshift interval.

Note that while sources are expected to also produce a strong Ly $\alpha$ line, because of internal reprocessing of ionizing photons into Ly $\alpha$ photons, these photons are not expected to have a significant effect. The photons stream into the $\mathrm{H}$ II region of their source, and by the time they reach the edge, many of them have redshifted above the Ly $\alpha$ wavelength by more than the line width; the remaining ones are trapped and diffuse only across a distance of approximately tens of parsecs before they too redshift out of the line. Thus, these photons only affect a very small fraction of the IGM, much less than the ionized fraction, which is itself small in the redshift range of interest here. Even though the Ly $\alpha$ flux within this volume can be high, the brightness temperature saturates when $x_{\text {tot }} \rightarrow \infty$. Observations on scales larger than the size of an $\mathrm{H}$ II region (which is $\lesssim 1$ comoving Mpc) smooth the signal over many such regions. The overall correction is thus small on both the mean brightness temperature and the fluctuations in it.

We therefore get (Madau et al. 1997)

$$
x_{\alpha}=\frac{16 \pi^{2} T_{*} e^{2} f_{\alpha}}{27 A_{10} T_{\gamma} m_{e} c} S_{\alpha} J_{\alpha},
$$

where $J_{\alpha}$ is the proper Ly $\alpha$ photon intensity (defined as the spherical average of the number of photons hitting a gas element per unit area per unit time per unit frequency per steradian) and $S_{\alpha}$ (Chen \& Miralda-Escudé 2004) is a correction factor between 1 and 2 that depends on the gas temperature and accounts for the redistribution of photon frequencies near the Ly $\alpha$ line center due to scattering off the thermal distribution of atoms. The intensity is itself given by a sum over $n$ (see also $\S 6$ ),

$$
J_{\alpha}=\frac{(1+z)^{2}}{4 \pi} \sum_{n=2}^{n_{\max }} \int_{z}^{z_{\text {max }}(n)} \frac{c d z^{\prime}}{H\left(z^{\prime}\right)} \epsilon\left(\nu_{n}^{\prime}, z^{\prime}\right),
$$

where absorption at level $n$ at redshift $z$ corresponds to an emitted frequency at $z^{\prime}$ of

$$
\nu_{n}^{\prime}=\nu_{\mathrm{LL}}\left(1-n^{-2}\right) \frac{\left(1+z^{\prime}\right)}{(1+z)},
$$

in terms of the Lyman limit frequency $\nu_{\mathrm{LL}}$. The comoving photon emissivity (defined as the number of photons emitted per 
unit comoving volume, per proper time and frequency, at restframe frequency $\nu$ at redshift $z$ ) is in turn

$$
\epsilon(\nu, z)=\bar{n}_{b}^{0} f_{*} \frac{d}{d t} F_{\mathrm{gal}}(z) \epsilon_{b}(\nu),
$$

where $\bar{n}_{b}^{0}$ is the cosmic mean baryon number density at $z=0$, $f_{*}$ is the efficiency with which gas is turned to stars in galactic halos, $F_{\text {gal }}(z)$ is the gas fraction in galaxies at $z$, and $\epsilon_{b}(\nu)$ is the spectral distribution function of the sources (defined as the number of photons per unit frequency emitted at $\nu$ per baryon in stars).

To determine the gas fraction in galaxies, we must first find the halo mass function. Although the basic model of Press \& Schechter (1974) describes the results of numerical simulations qualitatively, we use the halo mass function of Sheth \& Tormen (1999), which fits numerical simulations more accurately (Jenkins et al. 2001). In order to calculate fluctuations among large-scale regions of various densities in the global gas fraction that resides in galaxies within them, we must also find the halo mass function as a function of the overall mean density in each region. We adjust the mean halo distribution as a function of density based on the prescription of Barkana \& Loeb (2004), where we showed that this prescription fits a broad range of simulations including ones run at $z \sim 20$. As gas falls into a dark matter halo, it can fragment into stars only if its virial temperature is above $10^{4} \mathrm{~K}$ for cooling mediated by atomic transitions, or $\sim 500 \mathrm{~K}$ for molecular $\mathrm{H}_{2}$ cooling. Given a minimum cooling mass, we consider two possibilities for the star formation efficiency: either a fixed efficiency in all halos, as in equation (17), or an efficiency that drops as the square of the halo circular velocity $V_{c}$ at $V_{c} \lesssim$ $180 \mathrm{~km} \mathrm{~s}^{-1}$, as suggested by local observations (Dekel \& Woo 2003; Kauffman et al. 2003); in the latter case, we integrate over the halo mass function in order to determine the net effective value of $f_{*}$.

The spectral distribution function $\epsilon_{b}(\nu)$ depends on the assumed sources of radiation. In our discussion, we assume that stars dominate over miniquasars, as expected for the low-mass galaxies that are typical at high redshift (Wyithe \& Loeb 2004b). The stellar spectrum depends on the initial mass function (IMF) and the metallicity of the stars. In what follows, we consider two examples. The first is the locally measured IMF of Scalo (1998) with a metallicity of $1 / 20$ of the solar value, which we refer to as Population II stars. The second case, labeled as an extreme Population III IMF, consists entirely of zero-metallicity $M \gtrsim 100 M_{\odot}$ stars, as expected for the earliest generation of galaxies (Bromm $\&$ Larson 2004). In each case, we approximate the emissivity as a separate power law $\epsilon_{b}(\nu) \propto \nu^{\alpha_{S}-1}$ between every pair of consecutive levels of atomic hydrogen. For example, using Leitherer et al. (1999), we find that the Population II stars emit 9690 photons per baryon between Ly $\alpha$ and the Lyman limit, out of which 6520 photons are between $\operatorname{Ly} \alpha$ and $\operatorname{Ly} \beta$ with an $\alpha_{S}=0.14$ in this lowest frequency interval. The corresponding numbers for Population III stars (using Bromm et al. 2001) are 4800, 2670, and $\alpha_{S}=1.29$.

Figure 1 illustrates the cosmic evolution of the various key temperatures. The residual electron fraction keeps the gas temperature $T_{k}$ locked to the CMB temperature $\left[T_{\gamma}=2.725(1+z) \mathrm{K}\right]$ until $1+z \approx 142\left(\Omega_{b} h^{2} / 0.024\right)^{2 / 5}$ (Peebles 1993), but subsequently the gas cools adiabatically. The mean temperature offset $T_{b}$ is approximately $-30 \mathrm{mK}$ at $z=100$ and rises toward zero at low redshift until the Ly $\alpha$ flux from galaxies drops it again, to $-100 \mathrm{mK}$ and below. Note that this figure and our results in subsequent sections assume an adiabatically cooling

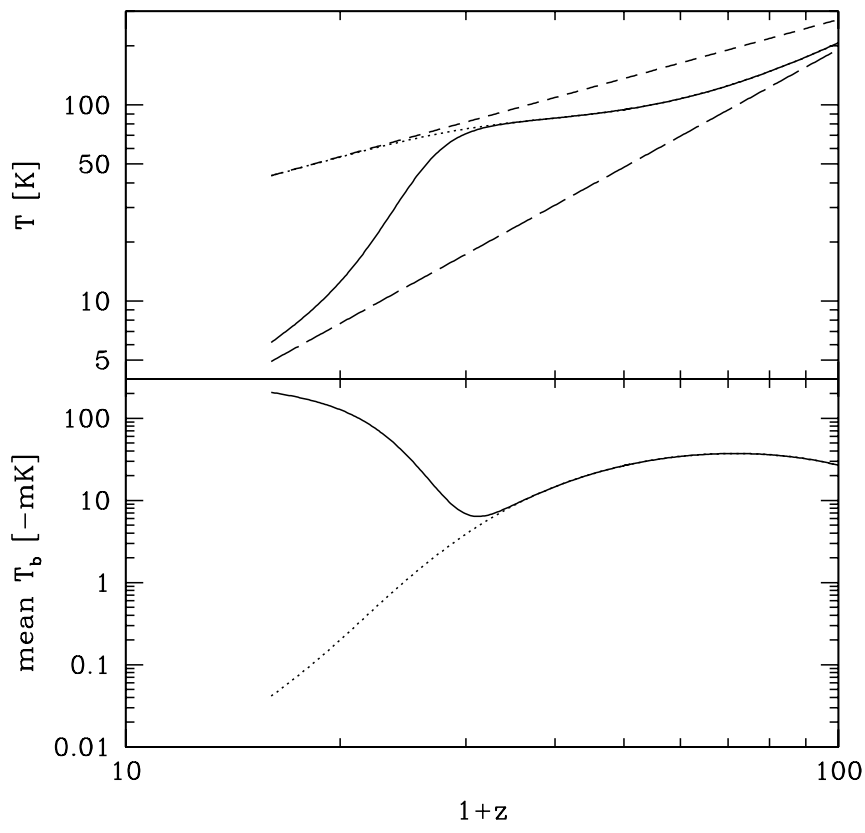

FIG. 1.-Redshift evolution of various mean temperatures. The mean $21 \mathrm{~cm}$ spin temperature is shown in the top panel for adiabatic IGM gas with Ly $\alpha$ coupling (solid line) and without it (dotted line). Also shown for comparison are the gas temperature (long-dashed line) and the CMB temperature (short-dashed line). The mean $21 \mathrm{~cm}$ brightness temperature offset relative to the $\mathrm{CMB}$ is shown in the bottom panel, with Ly $\alpha$ coupling (solid line) and without it (dotted line). The Ly $\alpha$ flux is calculated assuming that galaxies with Population III stars form in dark matter halos where the gas cools efficiently via atomic cooling. The star formation efficiency is normalized so that the Ly $\alpha$ coupling transition (i.e., $x_{\mathrm{tot}}=1$ ) occurs at redshift 20. [See the electronic edition of the Journal for a color version of this figure.]

cosmic gas, except where we explicitly assume preheating by X-rays.

\section{FLUCTUATIONS IN Ly $\alpha$ FLUX}

There are two separate sources offluctuations in the Ly $\alpha$ flux. The first is density inhomogeneities. Since gravitational instability proceeds faster in overdense regions, the biased distribution of rare galactic halos fluctuates much more than the global dark matter density (Kaiser 1984; Barkana \& Loeb 2004). When the number of sources seen by each atom is relatively small, Poisson fluctuations provide a second source offluctuations, statistically independent of the first source, to linear order. Unlike typical Poisson noise, these fluctuations are correlated between gas elements at different places, since two nearby elements see many of the same sources, where each source is at about the same distance from the two gas elements.

Since each hydrogen atom receives some Ly $\alpha$ flux from sources as far away as $\sim 250$ comoving Mpc, the flux is naively expected to be highly uniform based on the homogeneity of the universe on these large scales. However, the fluctuations in flux are actually relatively large because a significant portion of the flux comes from nearby sources. One reason is the $1 / r^{2}$ decline of flux with distance. In a homogeneous, nonexpanding universe, the flux per $\log r$ would scale linearly with $r$, since the $1 / r^{2}$ decline of the flux is overcome by the $r^{3}$ volume factor; while large scales would dominate the total flux, the relative contribution of small scales would already be far more significant than if we were simply counting galaxy numbers, where the overall scaling would be cubic instead of linear in $r$. The $1 / r^{2}$ scaling of flux also strongly magnifies Poisson noise since, e.g., the $21 \mathrm{~cm}$ emission along a given line of sight can fluctuate strongly owing to 
a single Ly $\alpha$ source that happens to lie very close to that line of sight. Although the mean flux at a point is well defined, if we calculate the mean squared flux at a point, then a shell at a distance $r$ contains a number of sources $\propto r^{2}$ and contributes to the squared flux a value $\propto 1 / r^{4}$; thus, the mean squared flux diverges at small radii, and since the correlation function of flux at $r=0$ is proportional to its mean square, it too diverges at small $r$.

A second reason for the dominance of small scales in the Ly $\alpha$ flux is that higher Lyman series photons, which are degraded to Ly $\alpha$ photons through scattering as discussed in the previous section, can only be seen from a small redshift interval that corresponds to the wavelength interval between two consecutive atomic levels of hydrogen. A third reason is that distant sources are observed after a time delay determined by the speed of light, and their relative contribution is reduced since galaxy formation was not as advanced at earlier times. To facilitate the weighting of different scales, we summarize equations (14), (15), and (17) in the form

$$
x_{\alpha}=\int_{z^{\prime}=z}^{z_{\max }(2)} \frac{d x_{\alpha}}{d r} \frac{d r}{d z^{\prime}} d z^{\prime}
$$

The top panel of Figure 2 shows an estimate of the relative contribution to the fluctuations in $\operatorname{Ly} \alpha$ flux from sources at various distances from a gas element at $z=20$. We take the contribution to the flux per $\log r$, i.e., $d x_{\alpha} / d \log r$ (which is $\propto r$ at small distances), and multiply it by the mean density fluctuation in spheres of radius $r$, in order to estimate the contribution per $\log r$ to the density-induced fluctuations in flux. We assume a scaleinvariant spectrum of primordial density fluctuations, as predicted by inflation and indicated by the latest observations (Seljak et al. 2005). Since this spectrum yields fluctuations that diverge only logarithmically at small scales, the declining contribution of small scales to the flux dominates as $r \rightarrow 0$. On the other hand, density fluctuations decrease as $r^{-2}$ on scales $r \gtrsim 100 \mathrm{Mpc}$, which overcomes the $\propto r$ contribution to the flux and reduces the role of large-scale modes in the flux fluctuations. Although these effects make the $\sim 10-100 \mathrm{Mpc}$ scales dominate the densityinduced fluctuations in $\operatorname{Ly} \alpha$ flux, the drop in the density of timeretarded sources (as evident from the comparison of the top two lines in the top panel) plus the large contribution of nearby sources through photons above $\operatorname{Ly} \beta$ make the overall effect smoothed out over scales of $\sim 0.1-100 \mathrm{Mpc}$. The figure shows discontinuous jumps at distances that correspond to the various redshifts $z_{\max }(n)$ that mark the Ly $\alpha$ horizons for photons emitted with energies between the $n \rightarrow 1$ and $n+1 \rightarrow 1$ transitions of hydrogen.

The bottom panel of Figure 2 shows the relative contribution to Poisson-induced fluctuations in the Ly $\alpha$ flux. We construct this estimate by multiplying $d x_{\alpha} / d \log r$ by the Poisson $1 / N^{1 / 2}$ fluctuation, where for small distances $N \propto r^{3}$. In a nonexpanding universe, this would already diverge as $1 / r^{1 / 2}$ at small scales. The previously mentioned effects only increase the dominance of small scales in the Poisson fluctuations. Thus, measuring the two different sources of flux fluctuations has a complementary effect and allows a broad range of scales to be probed. Furthermore, the Poisson fluctuations are independent (to linear order) of the underlying primordial density fluctuations and more directly measure the number density of sources. In Figure 2 and the following figures, we include in the case of "full Lyman band emission" all the photons emitted from Ly $\alpha$ up to a maximum range given by the interval between levels $n_{\max }$ and

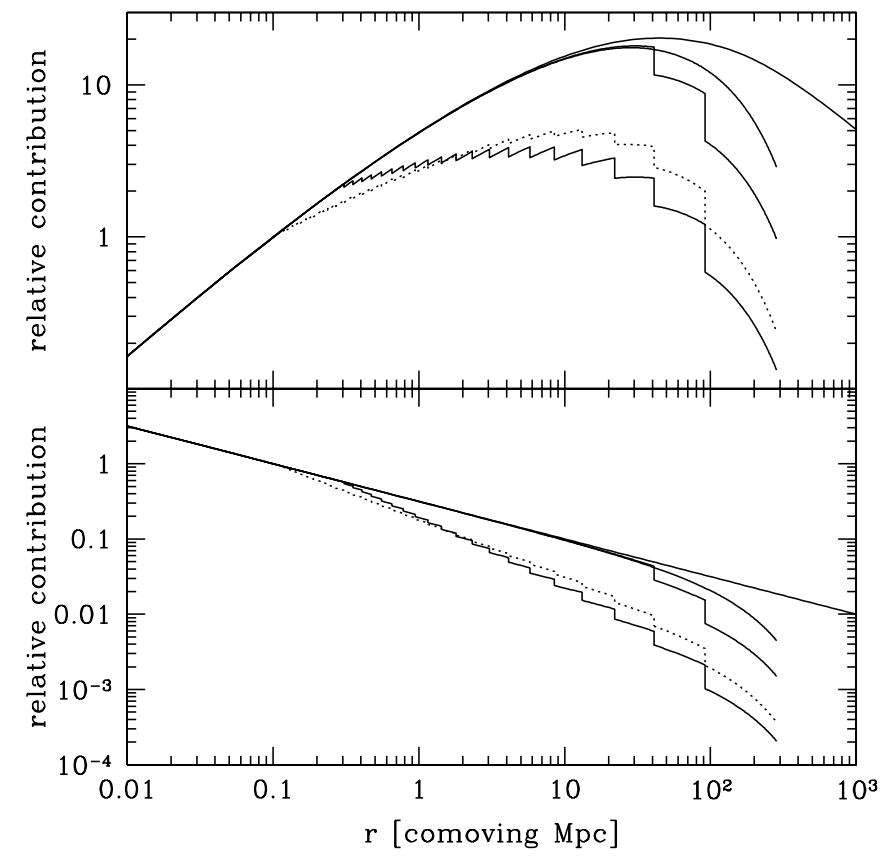

FIG. 2.- Relative contribution per $\log r$ of shells at various radii $r$ from a gas element at redshift $z=20$ to the fluctuations in the Ly $\alpha$ flux. We consider the fluctuations sourced by density inhomogeneities (top) and by Poisson fluctuations (bottom). In each set of solid lines, we show from top to bottom (at large $r$ ) the asymptotic case of a nonexpanding universe followed by galaxies, where we include stellar radiation emitted up to $\operatorname{Ly} \beta, \mathrm{Ly} \delta$, or the case of full Lyman band emission (see text). The minimum galaxy mass is set by the atomic cooling threshold in dark matter halos. Inside galaxies, we assume a fixed star formation efficiency and a Population III stellar population. Also shown for comparison is the case of full Lyman band emission with Population II stars (dotted line). All lines are arbitrarily normalized to unity at $r=0.1 \mathrm{Mpc}$. [See the electronic edition of the Journal for a color version of this figure.]

$n_{\max }+1$ of hydrogen (assuming that none of the photons are obscured or absorbed by dust within the host galaxy). We set this maximum level by cutting off photons that could only be seen from sources so nearby that the gas element would have to be within the $\mathrm{H}$ II region of the individual source, and thus this gas would be ionized and would not contribute to the $21 \mathrm{~cm}$ optical depth. Calculating a typical $\mathrm{H}$ II region size based on an escape fraction of ionizing photons of $f_{\text {esc }}=0.3$, this yields a maximum level $n_{\max }=23$ in the Population III case at $z=20$ and corresponds to including $99.3 \%$ of all photons emitted between Ly $\alpha$ and the Lyman limit. We note that since ionizing sources are clustered (Wyithe \& Loeb 2004b; Barkana \& Loeb 2004; Furlanetto et al. 2004b), the appropriate cutoff scale might be somewhat larger.

\section{POWER SPECTRUM OF FLUCTUATIONS IN THE $21 \mathrm{~cm}$ FLUX}

We first consider the effect of density perturbations on the flux as given by equation (18). When considering perturbations, we let $r$ be the Lagrangian position, which corresponds to the comoving distance at some initial high redshift. We now consider a field of perturbations $\delta_{0}(\boldsymbol{r})$, linearly extrapolated to the present from the initial time with the growth factor $D\left(z^{\prime}\right)$, so that the perturbations at a redshift $z^{\prime}$ are $\delta_{0}(\boldsymbol{r}) D\left(z^{\prime}\right)$. Consider the contribution of a Lagrangian volume $d V=d A d r$ at position $r$ to the flux observed at the origin of the coordinates at redshift $z$. The emission rate $\epsilon\left(\nu^{\prime}, z^{\prime}\right)$ is different than average, since the density perturbation $\delta_{0}(\boldsymbol{r}) D\left(z^{\prime}\right)$ changes the abundance of galaxies. On large scales, the change in the abundance of halos is 
linear in the density perturbation, for every halo mass (Mo \& White 1996; Barkana \& Loeb 2004), yielding a weighted bias $b\left(z^{\prime}\right)$ in the overall emission rate. Thus, the emission rate is increased by a factor $\left[1+b\left(z^{\prime}\right) \delta_{0}(\boldsymbol{r}) D\left(z^{\prime}\right)\right]$. Note that the required emitted frequency is determined by the redshift just as in the unperturbed case (i.e., eq. [16]), and also to first order we can use the unperturbed $z^{\prime}(r)$ relation in the perturbation term because corrections to this are small. The observed proper intensity at $z$ due to a proper emitting area $d A_{p}$ at the source at redshift $z^{\prime}$ is unchanged by perturbations, owing to the conservation of surface brightness known from gravitational lensing theory. However, for a fixed Lagrangian area $d A$, the proper area $d A_{p}$ (and thus also the observed intensity) is increased by the factor $[1+$ $\left.\frac{2}{3} \delta_{0}(\boldsymbol{r}) D\left(z^{\prime}\right)\right]$ to linear order. Finally, we must relate the remaining factor in the volume element $d V^{\prime}$, i.e., the radial width $d r$, to the redshift interval $d z^{\prime}$. The appropriate relation is $d z^{\prime}=\left(d v_{r}\right)$ $d r) d r / c$, where $v_{r}$ is the radial component of the physical velocity. The unperturbed form of this Doppler relation is $d z^{\prime}=$ $H\left(z^{\prime}\right) d r /\left[\left(1+z^{\prime}\right) c\right]$, which accounts for the denominator in equation (15). Even in the perturbed case, the radial integration limit is fixed in redshift as in equation (15). Note that we have assumed that the retarded time corresponding to a given source redshift $z^{\prime}>z$ is unperturbed; the perturbation in peculiar velocity causes a $\Delta z \sim \delta \mathrm{ar} /(\mathrm{c} / H)$, which is negligible compared to the biased galaxy fluctuation that corresponds to a $\Delta z \sim \delta(1+z)$ (Barkana \& Loeb 2004).

Integrating over the volume $d V^{\prime}$, we add the various terms mentioned before and obtain the total linear perturbation in $x_{\alpha}$, which is a spatial average of the density perturbation field, plus a spatial average of the velocity gradient term. The fractional perturbation in $d v_{r} / d r$ has a simple Fourier transform, $\tilde{\delta}_{d_{r} v_{r}}=$ $-\mu^{2} \tilde{\delta}$ (Kaiser 1987; Bharadwaj \& Ali 2004), which is used to separate the angular structure of the power spectrum (see $\S 3$ and Barkana \& Loeb 2005). Here $\mu$ is the cosine of the angle between the wavenumber $\boldsymbol{k}$ of the Fourier mode and the $\boldsymbol{r}$ (i.e., line of sight) direction. After proper averaging, we find the Fourier perturbation in $x_{\alpha}$ in terms of the Fourier transform of the density perturbation at $z$ :

$$
\tilde{\delta}_{\alpha}(\boldsymbol{k})=\tilde{W}(k) \tilde{\delta}(\boldsymbol{k}),
$$

where

$$
\begin{aligned}
& \tilde{W}(k) \\
& =\frac{1}{x_{\alpha}} \int_{z}^{z_{\max }(2)} d z^{\prime} \frac{d x_{\alpha}}{d z^{\prime}} \frac{D\left(z^{\prime}\right)}{D(z)}\left\{\left[1+b\left(z^{\prime}\right)\right] j_{0}(k r)-\frac{2}{3} j_{2}(k r)\right\},
\end{aligned}
$$

expressed in terms of spherical Bessel functions. The first term is reminiscent of the effective bias in galaxy number density, where the addition of 1 to the Lagrangian bias accounts for the higher physical density per unit volume. The second term is a geometrical correction for the case of flux, which arises from the velocity gradient term. The contribution to the power spectrum $P_{\alpha}(k)$ is then $\tilde{W}^{2}(k) P_{\delta}(k)$, while the contribution to $P_{\delta-\alpha}(k)$ is $\tilde{W}(k) P_{\delta}(k)$. We note that $P_{\alpha}$ corresponds to a real-space correlation function of the flux at two points $A$ and $B$ separated by a comoving distance $l$. In this correlation, if point $A$ is closer to sources at some location $\boldsymbol{r}$, then point $B$ will see the progenitors of the galaxies seen by point $A$. The relation between the flux of the progenitors and the flux of the later parent is im- plicitly accounted for in the above weighting by the redshiftdependent bias factor $b\left(z^{\prime}\right)$.

If the number of galaxies $N$ that contribute to the flux is relatively small, then in addition to fluctuations in the mean number density and mass distribution of halos, we expect significant Poisson fluctuations. We first derive a general framework for analyzing correlations induced by Poisson fluctuations. We note that in the context of the ionizing intensity due to quasars, Zuo (1992) derived the joint probability distribution of the intensities at two different points. Focusing on the two-point correlation function only, we present here a derivation that is simpler and more general, including cosmological redshift and time retardation effects.

We consider a large number of small volumes $d V$, such that the mean number of halos $\langle d n\rangle \ll 1$ in each, so that $d n^{2}=d n$ since at most a single halo may be found within a single volume element. Although we describe a volume distribution for convenience, this setup also applies to a generalized notion of volume, where the population of halos is divided up into many small units, and the effective position within this "volume" is specified by as many parameters as needed, in addition to spatial position (e.g., halo mass, emission time, merger history, etc.). We denote by $d x_{\alpha}^{A}$ the flux that arrives at a point $A$ due to $d n$ halos in some volume element; this flux is proportional to $d n$, with a proportionality factor that may depend on the distance to $A$ and on the various parameters that specify the volume element, and can incorporate all appropriate redshift effects. Then the mean expected flux $x_{\alpha}$ at a point $A$ is $\sum\left(d x_{\alpha}^{A} / d n\right)\langle d n\rangle$, while the expectation value of the flux at point $A$ times the flux at point $B$ is $\sum \sum\left(d x_{\alpha}^{A} / d n_{1}\right)\left(d x_{\alpha}^{B} / d n_{2}\right)\left\langle d n_{1} d n_{2}\right\rangle$. When the number of small volume elements is large, the double sum over different halos $d n_{1} \neq d n_{2}$ yields an expectation value $x_{\alpha}^{A} x_{\alpha}^{B}$, which is simply $x_{\alpha}^{2}$, while the sum over $d n_{1}=d n_{2}$ (which we then denote simply $d n$ ) yields the correlated Poisson term. Thus, the correlation function of relative Poisson fluctuations in flux is

$$
\xi_{\mathrm{P}}(l)=\frac{1}{x_{\alpha}^{2}} \sum \frac{d x_{\alpha}^{A}}{d n} \frac{d x_{\alpha}^{B}}{d n}\langle d n\rangle,
$$

where the points $A$ and $B$ are separated by a distance $l$.

To apply this equation, we divide halos into effective fourdimensional volumes $d V d M$, which include three spatial dimensions and a distribution in halo mass, and convert the sum into a four-dimensional integral. In a homogeneous, nonexpanding universe, the only difference between $d x_{\alpha}^{A} / d n$ and $d x_{\alpha}^{B} / d n$ would be the distance factors $1 / r_{A}^{2}$ and $1 / r_{B}^{2}$. For distant sources, $r_{A} \sim$ $r_{B} \gg l$, we have an integral $d V / r^{4}$ at all $r \gtrsim l$, so the contribution to $\xi_{\mathrm{P}}(l)$ of all such sources is $\propto 1 / l$. On the other hand, sources near $A$ satisfy $r_{A} \ll l$ and $r_{B} \approx l$, so their contribution is $d V /$ $\left(r_{A}^{2} l^{2}\right)$, over $r_{A}$ from 0 to $\sim l$, which is again $\propto 1 / l$. Sources near $B$ contribute equally, and the overall correlation function diverges at small separations as $\xi_{\mathrm{P}}(l) \propto 1 / l$, as expected from the simple scaling argument at the end of $\S 5$.

To apply this formalism in the present context, we first write the flux as a double integral over the halo number density as a function of mass and volume. Halos in a comoving volume $d V$ at $\boldsymbol{r}$ contribute a flux $\propto 1 / r^{2}$ at the origin, and we assume that the fraction of the flux contributed by halos of a given mass $M$ is proportional to the gas fraction contained in these halos. Thus, we write the contribution $d x_{\alpha}$ from the halos in $d V$ as

$$
d x_{\alpha} \equiv P\left(z^{\prime}\right) \frac{1}{r^{2}} \int_{M} M \frac{d n\left(z^{\prime}\right)}{d M} d M d V,
$$


where we have factored out explicitly the $1 / r^{2}$ dependence. Using the Sheth \& Tormen (1999) halo mass function $d n / d M$ at $z^{\prime}$, and $d x_{\alpha}$ calculated as in $\S 4$, the above equation defines $P\left(z^{\prime}\right)$ as an overall normalization factor that accounts for the spectral distribution and redshift effects. Note that the mean intensity $x_{\alpha}$ at a point is given by the volume integral of equation (22), which yields the same result as given by equation (18).

An additional subtlety necessitates a small adjustment in equation (21) before we can apply it in the present context. This equation, as written, requires that the same halos be seen by points $A$ and $B$ at the same redshift $z$. However, if, e.g., point $A$ is closer to some location $\boldsymbol{r}$, then the point $B$ will see the progenitors of the galaxies at $\boldsymbol{r}$ seen by point $A$. When we apply equation (21), we integrate over the halo distribution seen by the closer point $A$ and note that the total emission rate of its progenitors at the higher redshift $z_{B}^{\prime} \equiv z^{\prime}\left(r_{B}\right)$ is on average some fraction of the emission rate of the later parent galaxy at $z_{A}^{\prime} \equiv$ $z^{\prime}\left(r_{A}\right)$. An actual correlation function measured over a significantly large volume will indeed average over the progenitor distribution. This average fraction may depend on parent mass and the two redshifts. First, we neglect the dependence on parent mass; i.e., we consider a mass-averaged fraction. This is likely to yield an accurate result at high redshift, since when galaxies are rare they are all found in a narrow range of halo masses just above the threshold mass for star formation. We then fix the redshift dependence of this factor so that the total gas fraction in progenitors at $z_{B}^{\prime}$ is consistent with the total fraction calculated in the normal way from the halo mass function at $z_{A}^{\prime}$. Thus, the dimensionless correlation function is

$$
\xi_{\mathrm{P}}(l)=\frac{2}{x_{\alpha}^{2}} \int_{V} d V \int_{M} \frac{d n\left(z_{A}^{\prime}\right)}{d M} d M M^{2} \frac{P\left(z_{A}^{\prime}\right)}{r_{A}^{2}} \frac{P\left(z_{B}^{\prime}\right)}{r_{B}^{2}} \frac{F_{\mathrm{gal}}\left(z_{B}^{\prime}\right)}{F_{\mathrm{gal}}\left(z_{A}^{\prime}\right)},
$$

where $d V=d^{3} r_{A}$, and we integrate over half the volume where $r_{A}<r_{B}$, with the factor of 2 accounting for the contribution of sources that are closer to $B$.

We have assumed in calculating $r_{B}$ that the progenitors of a galaxy are at the same location as the parent. The actual displacements are at most of order the Lagrangian size of a typical galactic halo, $\sim 0.1$ comoving $\mathrm{Mpc}$, which is negligible when $r_{B} \gg 0.1 \mathrm{Mpc}$, while if we consider small values of $r_{B}$, then the displacements resulting from gravitational infall velocities $(\leqslant c /$ 1000) during the time interval $\left|r_{B}-r_{A}\right| / c$ are still a small fraction of the distance $r_{B}-r_{A}$.

The correlation function $\xi_{\mathrm{P}}(l)$ drops sharply at large distances, and in particular, when $l$ is more than twice the distance corresponding to $z_{\max }(2)$, no halo can contribute flux to both points $A$ and $B$ and thus $\xi_{\mathrm{P}}(l)=0$. At small distances, $\xi_{\mathrm{P}}(l) \propto 1 / l$ as in a nonexpanding universe, but even for $l \rightarrow 0$ the proportionality factor is affected by distant sources since they dominate the flux (compare Zuo 1992). Figure 3 illustrates this correlation function in several cases. The maximum $\operatorname{Ly} \alpha$ horizon in this case, corresponding to the redshift $z_{\max }(2)$, is 284 comoving Mpc. When we include only the stellar radiation emitted up to $\mathrm{Ly} \beta$, $\xi_{\mathrm{P}}(l)$ begins to drop below the $1 / l$ asymptotic form at separations $l \gtrsim 200 \mathrm{Mpc}$ and sharply drops to zero at twice the Ly $\alpha$ horizon. However, when more energetic radiation is included, $\xi_{\mathrm{P}}(l)$ drops below the $1 / l$ power law at much smaller $l$, corresponding to the smaller effective source horizons of the energetic photons, thus making the cutoff at $l \sim 200 \mathrm{Mpc}$ much rounder and less prominent.

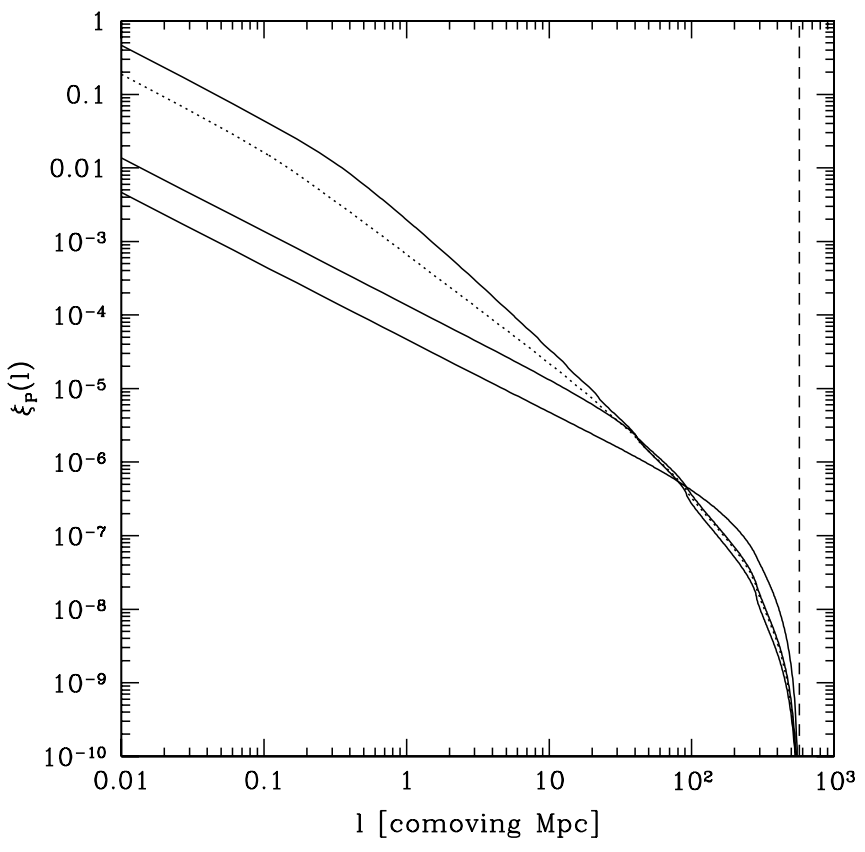

FIG. 3.-Poisson-induced dimensionless correlation function of $21 \mathrm{~cm}$ brightness fluctuations vs. comoving separation. We assume that galaxies formed via atomic cooling in halos at $z=20$ with a fixed star formation efficiency in all halos, set to produce the coupling transition at this redshift (so that $x_{\text {tot }}=1$ ). We also assume that the IGM gas has been cooling adiabatically down to this redshift. In each set of solid lines we include, from bottom to top at $l=1 \mathrm{Mpc}$, stellar radiation emitted up to $\operatorname{Ly} \beta, \operatorname{Ly} \delta$, or full Lyman band emission ( $\operatorname{see} \oint 5$ ), assuming Population III stars. Also shown for comparison is the case of full Lyman band emission with Population II stars (dotted line). We also show the separation equal to twice the maximum Ly $\alpha$ horizon (dashed line). [See the electronic edition of the Journal for a color version of this figure.]

In this figure, the star formation parameters are fixed to produce the Ly $\alpha$ coupling transition (i.e., $x_{\text {tot }}=1$ ) at $z=20$. In our standard case of full Lyman band emission and Population III stars, with only atomic cooling occurring in galactic halos, the required star formation efficiency is relatively low, $0.22 \%$, and $f_{\text {esc }}=0.3$ yields an ionized fraction of $1 \%$ at $z=20$, with complete reionization at $z=9$. Although here we have calculated $\xi_{\mathrm{P}}(l)$, in the rest of the paper we Fourier transform the Poisson-induced correlation function and find the corresponding power spectrum.

For the case of a mass-dependent star formation efficiency, we insert the appropriate weighting factor into the various relations given in this section. Note that if star formation in individual galaxies actually proceeded in short, random bursts with a duty cycle $\eta \ll 1$, then the higher rate of star formation during bursts (by a factor $1 / \eta$ ) would counterbalance the fraction of time of burst activity $(\eta)$, and the overall correlation function would be unchanged for both of the sources that we consider for fluctuations in flux.

We insert two additional cutoffs that are expected on small scales. The first is the cutoff due to baryonic pressure, which affects the density fluctuations and has an approximate shape $(1+$ $\left.k^{2} R_{F}^{2}\right)^{-2}$ (Shapiro et al. 1994; Gnedin \& Hui 1998), where the filtering scale is $R_{F}=2.9\left(M_{F} / 10^{6} M_{\odot}\right)^{1 / 3}\left(\Omega_{m} h^{2} / 0.14\right)^{-1 / 3} \mathrm{kpc}$, in terms of the filtering mass $M_{F}$, which is a weighted time average of the Jeans mass (Gnedin \& Hui 1998). We calculate $M_{F}$ appropriately for the two cases we consider, an adiabatically cooling IGM or a preheated IGM. The second cutoff that we include results from the thermal width of the $21 \mathrm{~cm}$ line, which also smooths out the fluctuations on small scales. We approximate 


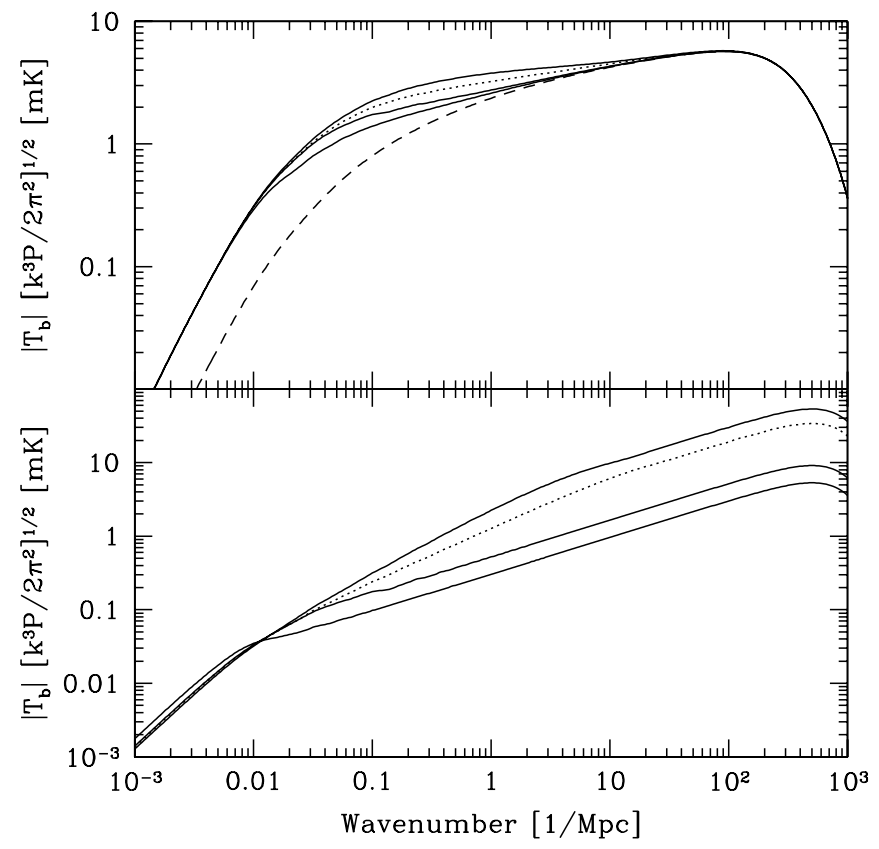

FIG. 4.-Power spectra of $21 \mathrm{~cm}$ brightness fluctuations vs. comoving wavenumber. Shown are $P_{\mu^{2}}$ (top), which contains the contribution of the densityinduced fluctuations in flux, and $P_{\text {un }}-\delta$ (bottom), which is solely due to the Poisson-induced fluctuations. We assume that galaxies formed via atomic cooling in halos at $z=20$ with a fixed star formation efficiency in all halos, set to produce the coupling transition at this redshift (so that $x_{\mathrm{tot}}=1$ ). We also assume that the IGM gas has been cooling adiabatically down to this redshift. In each set of solid lines we include, from bottom to top at $k=0.1 \mathrm{Mpc}^{-1}$, stellar radiation emitted up to $\operatorname{Ly} \beta, \operatorname{Ly} \delta$, or full Lyman band emission (see $\S 5$ ), assuming Population III stars. Also shown for comparison is the case of full Lyman band emission with Population II stars (dotted line). We also show $2 \beta P_{\delta}$ (dashed line). [See the electronic edition of the Journal for a color version of this figure.]

this effect as a Gaussian cutoff, $e^{-k^{2} R_{T}^{2}}$, where the spatial scale corresponding to the one-dimensional thermal velocity distribution is

$$
\begin{aligned}
R_{T}= & 7.0\left(\frac{m_{p}}{1.22}\right)^{-1 / 2}\left(\frac{T_{k}}{100 \mathrm{~K}}\right)^{1 / 2} \\
& \times\left(\frac{1+z}{10}\right)^{-1 / 2}\left(\frac{\Omega_{m} h^{2}}{0.14}\right)^{-1 / 2} \mathrm{kpc}
\end{aligned}
$$

where $m_{p}$ is the mean mass per particle of neutral primordial gas in units of the proton mass.

For the same parameters as in Figure 3, Figure 4 shows the $\mu^{2}$ angular component of the observable power spectra, given by

$$
P_{\mu^{2}}(k)=2 P_{\delta}(k)\left[\beta+\frac{x_{\alpha}}{\tilde{x}_{\mathrm{tot}}} \tilde{W}(k)\right]
$$

and $P_{\text {un }-\delta}(k)$, which equals the Poisson power spectrum except for a factor of $\left(x_{\alpha} / \tilde{x}_{\text {tot }}\right)^{2}$. In addition to these quantities, $P_{\mu^{0}}$ yields the baryonic density power spectrum at each redshift, and its normalization (measured in $\mathrm{mK}$ ) thus yields the mean brightness temperature $T_{b}$. Note that the actual plotted quantity is $\left|T_{b}\right|\left[k^{3} P(k) /\left(2 \pi^{2}\right)\right]^{1 / 2}$, i.e., the square root of the contribution per $\log k$ of power on the scale $k$ to the variance of the brightness temperature. The figure shows that $\beta$ can be determined from $P_{\mu^{2}} / P_{\mu^{4}}$ on small scales $\left(k \gtrsim 1 \mathrm{Mpc}^{-1}\right)$, making the two cases (adiabatic: $\beta \lesssim \frac{1}{3}$, or preheated: $\beta \approx 1$ ) easily distinguishable. As a result, the mean brightness temperature determines $x_{\text {tot }}$ in these two limits (see eq. [4]). Measurements at $k \gtrsim 100 \mathrm{Mpc}^{-1}$ can also independently probe $T_{k}$ because of the smoothing effects of the gas pressure and the thermal width of the $21 \mathrm{~cm}$ line.

Note that the value of $x_{\alpha}$ (which dominates $x_{\text {tot }}$ in the cases shown in Fig. 4) is interesting in itself, since it fixes the product of the fraction of gas in galaxies times the typical efficiency with which gas is turned to stars and the stars produce ultraviolet photons inside these galaxies. The figure also shows that the power spectra are strongly sensitive to the radial distribution of flux seen by the gas, which in turn probes the contribution of photons from the higher level Lyman series, a contribution that depends on the stellar IMF and the spectrum of the sources. In particular, since $\xi_{\mathrm{P}}(l)$ drops to zero at large $l$, the corresponding Poissoninduced power spectrum approaches a constant at small $k$, and the plotted quantity is $\propto k^{3 / 2}$.

$\mathrm{X}$-ray heating of the cosmic gas above the CMB temperature is likely to occur well before the universe is ionized because it requires an amount of energy of only $\sim 5 \times 10^{-3}[(1+z) / 20] \mathrm{eV}$ per baryon, while reionization requires $\sim 13.6 f_{\text {esc }}^{-1} \mathrm{eV}$ per hydrogen atom (or even more if recombinations are included). The X-rays may be produced by stellar winds, supernovae, X-ray binaries, quasars, or collapsed halos (see Madau et al. 1997 and $\S 2.3 .2$ in Wyithe \& Loeb 2004a). As long as more than $\sim 0.1 \%$ of the energy radiated by galaxies above the Lyman limit couples to the IGM through X-ray scattering, $T_{k}$ exceeds $T_{\gamma}$ before the completion of reionization. It is possible, however, that Ly $\alpha$ coupling between $T_{s}$ and $T_{k}$ is achieved before X-rays heat $T_{k}$ above $T_{\gamma}$.

We note that a significant gas fraction may already lie in virialized minihalos at high redshift. Although this gas may be dispersed owing to internal feedback from star formation due to molecular hydrogen cooling (Oh \& Haiman 2003; Ricotti et al. 2002), if stars cannot form, then the dense gas minihalos produce $21 \mathrm{~cm}$ fluctuations (Iliev et al. 2002, 2003). Since the gas content is strongly suppressed in halos below the filtering mass (Gnedin 2000), we estimate the maximum gas fraction in minihalos as lying between the filtering mass $M_{F}$ and the minimum mass needed for atomic cooling. At $z=20$, only around $0.6 \%$ of the gas is in minihalos in the mass range $8 \times 10^{5}-3 \times 10^{7} M_{\odot}$, compared to $11 \%$ in minihalos in the range $4 \times 10^{5}-1 \times 10^{8} M_{\odot}$ at $z=8$. Thus, in the redshift range that we focus on in this paper, only a minor fraction of the gas is in virialized regions and so we consider the density and temperature distributions only in uncollapsed regions.

\section{STUDYING THE EARLIEST GALAXIES}

Observing the enhanced $21 \mathrm{~cm}$ fluctuations would constitute a direct detection of the collective emission of the earliest generation of galaxies, long before reionization. Such a detection is potentially feasible within a few years, ${ }^{3}$ while the number of stars in such an early galaxy may be smaller than in a presentday globular cluster, making these galaxies at $z \sim 20-30$ undetectable individually even with the proposed James Webb Space Telescope $e^{4}$ planned for launch in the next decade.

In order to illustrate how the properties of the first galaxies may be probed with $21 \mathrm{~cm}$ fluctuations, we predict the observed

\footnotetext{
3 See http://space.mit.edu/eor-workshop.

4 See http://www.jwst.nasa.gov.
} 


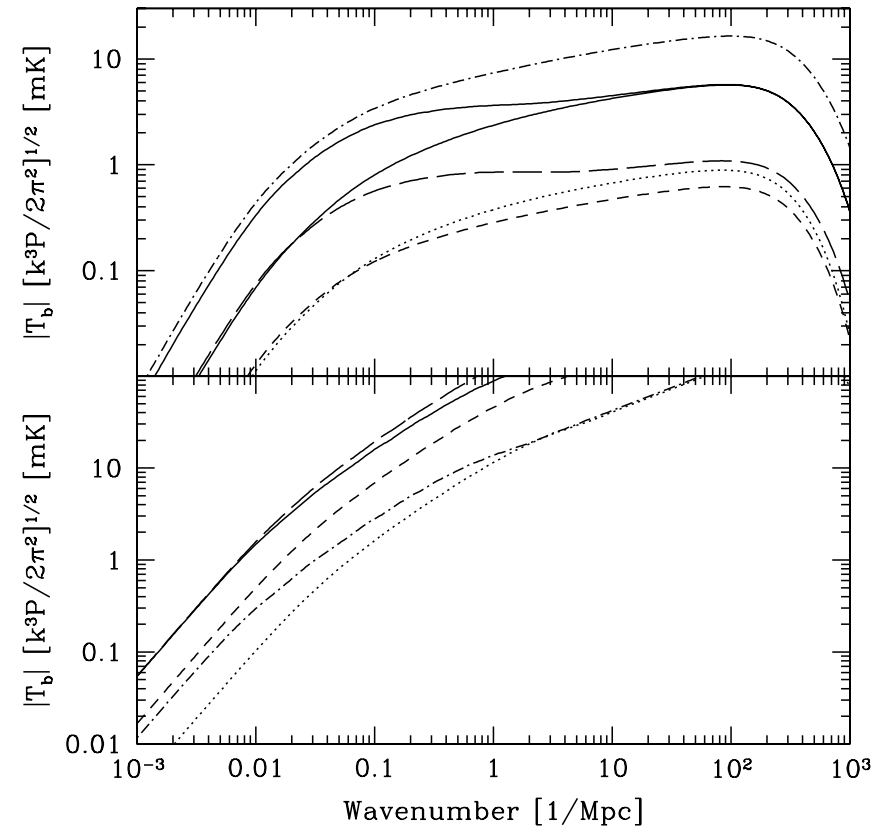

FIG. 5.-Power spectra of $21 \mathrm{~cm}$ brightness fluctuations vs. comoving wavenumber. Shown are $P_{\mu^{2}}$ (top), which contains the contribution of the densityinduced fluctuations in flux, and $P_{\mathrm{un}}-\delta$ (bottom), which is solely due to the Poisson-induced fluctuations. We assume atomic cooling with an efficiency that varies according to local observations (see text), normalized so that $x_{\text {tot }}=1$ at $z=20$. Redshifts shown are $z=35$ (dotted line), 30 (short-dashed line), 25 (long-dashed line), 20 (upper solid line), and 15 (dot-dashed line). We also show $2 \beta P_{\delta}$ at $z=20$ (top panel, lower solid line). We assume full Lyman band emission (see $\S 5$ ) and Population III stars. [See the electronic edition of the Journal for a color version of this figure.]

power spectra at a sequence of redshifts, for various choices of the parameters of galactic halos and IGM heating. Figure 5 shows a reasonable lower limit on the number of halos in the universe. Here we maximize the dominance of massive halos by assuming that (1) atomic cooling is required for efficient star formation and (2) the star formation efficiency declines with circular velocity according to local observations (Dekel \& Woo 2003; Kauffman et al. 2003), emphasizing the role of high-mass halos. The star formation efficiency is set to $12 \%$ above $V_{c}=180 \mathrm{~km}$ $\mathrm{s}^{-1}$ and $\propto V_{c}^{2}$ below this value. In this case, reionization completes at $z=11.7$. In Figure 5 the threshold parameter $x_{\text {tot }}$ (going from high redshift to low) is $0.070,0.037,0.12,1.0$, and 6.2. The corresponding value of $\beta$ is $2.1,2.0,0.36,0.22$, and 0.25 , respectively. We find at $z=20$ a filtering mass $M_{F}=$ $8.0 \times 10^{5} M_{\odot}, R_{F}=2.7 \mathrm{kpc}$, and $R_{T}=1.4 \mathrm{kpc}$. Collisions dominate the Ly $\alpha$ coupling at $z \gtrsim 30$ (contributing $x_{c}=0.070,0.029$, and 0.008 at the three highest redshifts shown), producing a high $\beta$ and suppressing the effect of the fluctuations in Ly $\alpha$ flux, which are largest at the highest redshifts. The absolute signal in $\mathrm{mK}$ tends to rise with time owing to the growth of density fluctuations (which affects $P_{\mu^{2}}$ ) and the declining gas temperature, which increases the overall $21 \mathrm{~cm}$ absorption in the adiabatic case. However, the increasing number density of sources with time eventually suppresses the Poisson fluctuations. Also, the saturation of the Ly $\alpha$ coupling (i.e., $x_{\text {tot }} \gg 1$ ) reduces the sensitivity of the $21 \mathrm{~cm}$ fluctuations to fluctuations in the Ly $\alpha$ flux; in the figure, by $z=15$ the power spectrum $P_{\mu^{2}}(k)$ has essentially reverted back to the shape of $P_{\delta}(k)$. The figure does not include the signature of ionized bubbles, which becomes significant only at later times when the ionized fraction approaches unity.

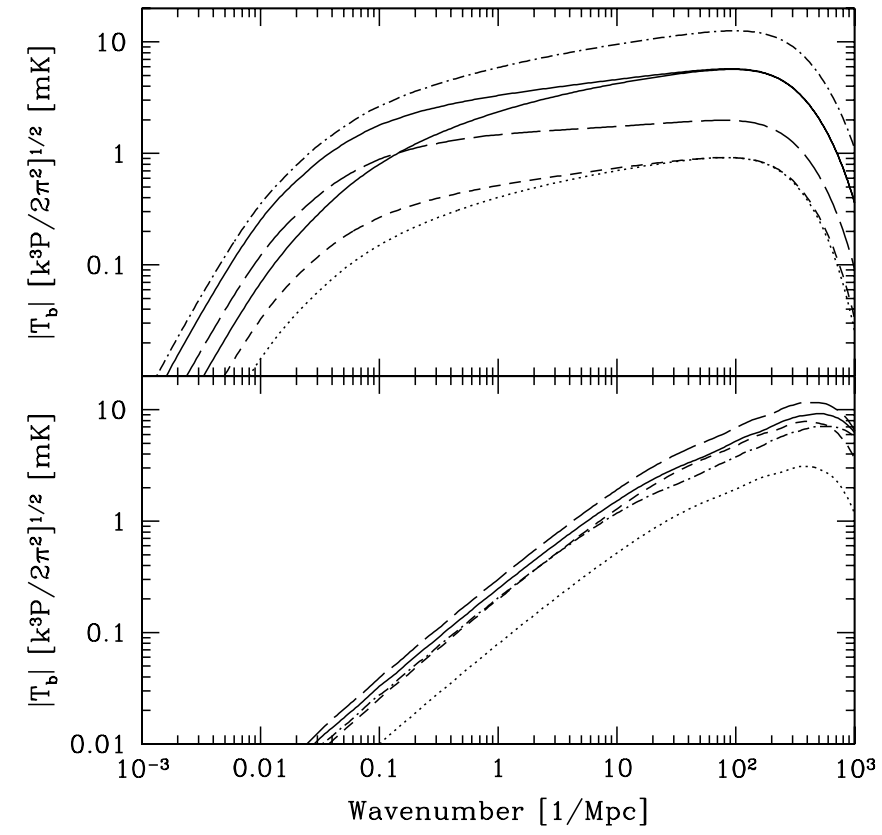

FIG. 6.-Power spectra of $21 \mathrm{~cm}$ brightness fluctuations vs. comoving wavenumber. Shown are $P_{\mu^{2}}$ (top), which contains the contribution of the densityinduced fluctuations in flux, and $P_{\mathrm{un}}-\delta$ (bottom), which is solely due to the Poisson-induced fluctuations. We assume $\mathrm{H}_{2}$ cooling with a fixed efficiency set so that $x_{\text {tot }}=1$ at $z=20$. Redshifts shown are $z=35$ (dotted line), 30 (shortdashed line), 25 (long-dashed line), 20 (upper solid line), and 15 (dot-dashed line). Note the different axis ranges compared to Fig. 5. We also show $2 \beta P_{\delta}$ at $z=20$ (top panel, lower solid line). We assume full Lyman band emission (see $\S 5$ ) and Population III stars. [See the electronic edition of the Journal for a color version of this figure.]

Figure 6 shows a reasonable upper limit on the number of halos in the universe. In this case we maximize the contribution of numerous low-mass halos by assuming that only molecular cooling is required for efficient star formation, with a fixed star formation efficiency $\left(f_{*}=0.03 \%\right)$. With these parameters, the universe would not complete reionization by redshift 6 without a significant rise in $f_{*}$ at $z \gtrsim 6$. Figure 6 shows some of the same trends that are apparent in the previous figure, although both sources of fluctuations in flux are significantly smaller, since there are more halos contributing and they are more weakly biased. The threshold parameter $x_{\text {tot }}$ (going from high redshift to low) is 0.076 , $0.082,0.30,1.0$, and 1.9. As before, collisions dominate at high redshift, contributing $x_{c}=0.070,0.029$, and 0.008 at the three highest redshifts shown. The values of $\beta$ are $1.9,0.95,0.24$, 0.22 , and 0.25 , respectively.

In Figure 7 we consider the same halo population as in Figure 5, but with the $21 \mathrm{~cm}$ fluctuations modified owing to an assumed preheating of the IGM. We set the star formation efficiency to $6.9 \%$ above $V_{c}=180 \mathrm{~km} \mathrm{~s}^{-1}$ and $\propto V_{c}^{2}$ below this threshold, so that $x_{\text {tot }}=1$ at $z=20$. The values of $x_{\alpha}$ at the various redshifts are $0.0003,0.006,0.08,0.79$, and 5.3, while $x_{c}=0.61,0.46$, $0.32,0.21$, and 0.12 , respectively. In this case, collisions are still significant at the lower redshifts because of the increased collision rate at high temperature. The value of $\beta$ is $1.6,1.7,1.6$, 1.1 , and 1.0 , respectively, always $\gtrsim 1$ since the gas is uniformly heated and no longer changes its temperature adiabatically. In this case $M_{F}=2.4 \times 10^{6} M_{\odot}, R_{F}=3.9 \mathrm{kpc}$, and $R_{T}=10.8 \mathrm{kpc}$ at $z=20$. Comparing Figures 5 and 7 , we see that the strong differences between these two cases in both the mean $T_{b}$ and the value of $\beta$ (which affects $P_{\mu^{2}}$ ) allow for a consistency check on the thermal state of the IGM. Note that in the cases shown in 


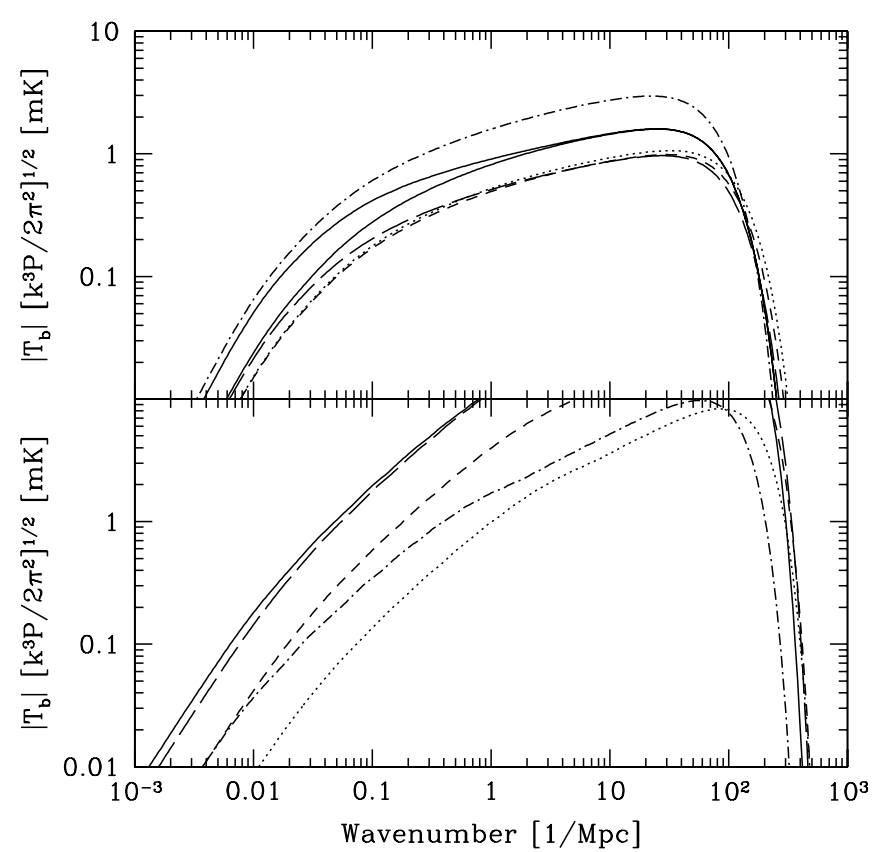

FIG. 7.- Power spectra of $21 \mathrm{~cm}$ brightness fluctuations vs. comoving wavenumber. Same as Fig. 5, except that we assume preheating to $500 \mathrm{~K}$ by $\mathrm{X}$-ray sources. Note the different axis ranges compared to Fig. 5. [See the electronic edition of the Journal for a color version of this figure.]

Figures 5 and 7, the Poisson fluctuations diverge on small scales, in which case our linear analysis based on equation (7) represents an estimate that is subject to strong nonlinear corrections. Also, on scales $\lesssim 0.1$ comoving Mpc, which corresponds to the initial region that collapses to become a typical halo at $z \sim 20$, our calculation is subject to corrections owing to nonlinear gravitational collapse.

From the ratio $P_{\mu^{2}} / P_{\mu^{4}}$ at large scales, it is possible to measure $\tilde{W}(0)$ (see eq. [25]), which yields directly the average bias factor of the emitting sources, given by equation (20) with the angle brackets becoming simply $\left[1+b\left(z^{\prime}\right)\right]$ in this limit. The relative bias of galaxies is on large scales predicted to depend simply on the rarity (number of sigma) of the underlying Gaussian density fluctuations that led to their formation. Thus, it is sensitive to the typical mass of the dark matter halos that host galaxies at these redshifts. This large-scale enhancement factor can be easily seen by comparing the upper solid line to the dashed line in Figure 4, as well as the pair of $z=20$ lines in each of Figures 5, 6, and 7. The enhancement factor shown in the figure (which equals the square root of the ratio between $P_{\mu^{2}}$ and $\left.2 \beta P_{\delta}\right)$ is in each case $4.8,5.1,4.0$, and 2.2 , respectively. The corresponding value of $\tilde{W}(0)$ is $9.8,11.2,6.5$, and 11.2 , respectively.

\section{SUMMARY}

We have shown that the $21 \mathrm{~cm}$ flux fluctuations from intergalactic hydrogen are strongly enhanced owing to the radiation emitted by the first galaxies at redshifts $\sim 20-30$. The earliest galaxies emitted photons with energies between the Ly $\alpha$ and Lyman limit transitions of hydrogen, to which the neutral universe was transparent except at the Lyman series resonances. As these photons redshifted into a Lyman series resonance and then degraded into an Ly $\alpha$ photon, they coupled the spin temperature $T_{S}$ of the $21 \mathrm{~cm}$ transition of hydrogen to the gas ki- netic temperature $T_{k}$, allowing it to deviate from the microwave background temperature, $T_{\gamma}$. Figures 4-7 show that the fluctuations in the radiation emitted by the first galaxies enhanced the overall power spectrum of $21 \mathrm{~cm}$ flux fluctuations by at least a factor of a few during the period when Ly $\alpha$ coupling was established. The enhancement is sourced by inhomogeneities in the biased distribution of galaxies (as those represent rare peaks in the density field), as well as by Poisson noise in the number of galaxies. The effects of galaxies are more apparent when the power spectrum is split into angular components relative to the line of sight (with the anisotropy sourced by peculiar velocities); in particular, the component $P_{\text {un }-\delta}$ shown in the bottom panels of Figures 4-7 (eq. [11]) would vanish in the absence of Poisson fluctuations in the distribution of galaxies. For the density-induced fluctuations (top panels), the enhancement factor is roughly constant on scales $\gtrsim 10$ comoving Mpc. Because of the rapid evolution in the abundance of galaxies at early cosmic times and the atomic physics of resonant scattering by hydrogen, the fluctuations in the $\operatorname{Ly} \alpha$ intensity are dominated by the contribution from sources within distances $\sim 1-100$ comoving Mpc, well within the source horizon out to which an $\mathrm{Ly} \beta$ photon could redshift into an Ly $\alpha$ photon (Fig. 2).

Several experiments are currently being constructed or designed to detect the $21 \mathrm{~cm}$ fluctuations prior to reionization. For an overview, see the Web site for the MIT Epoch of Reionization Workshop; ${ }^{5}$ for individual experiments, see the LOFAR, ${ }^{6}$ MWA, ${ }^{7}$ PAST, ${ }^{8}$ and the future SKA ${ }^{9}$ Web sites. Although lowfrequency foregrounds are much brighter than the $21 \mathrm{~cm}$ signal, they are not expected to include sharp spectral features, unlike the $21 \mathrm{~cm}$ maps that should decorrelate over small shifts in frequency (corresponding to slicing the universe at different redshifts). Consequently, the prospects for extraction of the $21 \mathrm{~cm}$ signal are promising (Gnedin \& Shaver 2004; Zaldarriaga et al. 2004; Morales \& Hewitt 2004; Morales 2005; Santos et al. 2004). The signal-to-noise ratio in these experiments is controlled by the sky brightness and grows as the square root of the integration time. Hence, the increase in the amplitude of $21 \mathrm{~cm}$ fluctuations that we predict has important practical implications for observing the $21 \mathrm{~cm}$ signal from the corresponding redshifts, since the required integration time is reduced by the square of the enhancement factor.

The enhanced level of fluctuations in the $21 \mathrm{~cm}$ flux lasted only until the Ly $\alpha$ coupling of $T_{s}$ and $T_{k}$ was saturated. Both before and after the Ly $\alpha$ coupling epoch, the enhancement (compared to the case of a uniform Ly $\alpha$ flux) had a lower amplitude (see, e.g., Fig. 5, where Ly $\alpha$ coupling was achieved at $z \sim 20$ ). Detection of the enhancement factor over the available redshift interval (i.e., the corresponding observed frequency band) can be used to infer the abundance and characteristic mass of the earliest galaxies $(\S 7)$ without any such galaxy being observed individually. The existence of Ly $\alpha$ coupling can also be used to determine whether $T_{k}$ is larger than $T_{\gamma}$ in this redshift interval. If the $21 \mathrm{~cm}$ signal appears in emission rather than in absorption (where absorption is present in the interval $30 \lesssim$ $z \lesssim 200$; Loeb \& Zaldarriaga 2004), then $T_{k}>T_{\gamma}$, most likely as a result of heating from $\mathrm{X}$-rays emitted by the same galaxies.

\footnotetext{
5 Available at http://space.mit.edu/eor-workshop.

6 Available at http://www.lofar.org.

7 Available at http://web.haystack.mit.edu/arrays/MWA/index.html.

8 Available at http://xxx.lanl.gov/abs/astro-ph/0404083.

9 Available at http://www.skatelescope.org.
} 
Altogether, the $21 \mathrm{~cm}$ fluctuations can be used to probe the level of UV and X-ray radiation emitted by the earliest galaxies, long before reionization was complete. The milestones of Ly $\alpha$ coupling between $T_{s}$ and $T_{k}$ and X-ray heating of $T_{k}$ above $T_{\gamma}$ provide important precursors for the radiative feedback of galaxies on the IGM that culminated with the completion of reionization.
We acknowledge support by NSF grant AST 02-04514. R. B. is grateful for the kind hospitality of the Harvard-Smithsonian CfA and acknowledges the support of NSF grant PHY99-07949 at KITP, Israel Science Foundation grant 28/02/01, and an Alon Fellowship at Tel Aviv University. This work was also supported in part by NASA grant NAG 5-13292 and NSF grants AST 0071019 (for A. L.).
Allison, A. C., \& Dalgarno, A. 1969, ApJ, 158, 423

Barkana, R., \& Loeb, A. 2001, Phys. Rep., 349, 125 2004, ApJ, 609, 474 2005, ApJ, 624, L65

Bharadwaj, S., \& Ali, S. S. 2004, MNRAS, 352, 142

Bromm, V., Kudritzki, R. P., \& Loeb, A. 2001, ApJ, 552, 464

Bromm, V., \& Larson, R. 2004, ARA\&A, 42, 79

Chen, X., \& Miralda-Escudé, J. 2004, ApJ, 602, 1

Ciardi, B., \& Madau, P. 2003, ApJ, 596, 1

Dekel, A., \& Woo, J. 2003, MNRAS, 344, 1131

Field, G. B. 1958, Proc. IRE, 46, 240

Furlanetto, S. R., Zaldarriaga, M., \& Hernquist, L. 2004a, ApJ, 613, 1 2004b, ApJ, 613, 16

Gnedin, N. Y. 2000, ApJ, 542, 535

Gnedin, N. Y., \& Hui, L. 1998, MNRAS, 296, 44

Gnedin, N. Y., \& Shaver, P. A. 2004, ApJ, 608, 611

Hogan, C. J., \& Rees, M. J. 1979, MNRAS, 188, 791

Iliev, I. T., Scannapieco, E., Martel, H., \& Shapiro, P. R. 2003, MNRAS, 341, 81

Iliev, I. T., Shapiro, P. R., Ferrara, A., \& Martel, H. 2002, ApJ, 572, L123

Jenkins, A., et al. 2001, MNRAS, 321, 372

Kaiser, N. 1984, ApJ, 284, L9 1987, MNRAS, 227, 1

Kauffman, G., et al. 2003, MNRAS, 341, 54

Leitherer, C., et al. 1999, ApJS, 123, 3

Loeb, A., \& Zaldarriaga, M. 2004, Phys. Rev. Lett., 92, 211301

\section{REFERENCES}

Madau, P., Meiksin, A., \& Rees, M. J. 1997, ApJ, 475, 429

Mo, H. J., \& White, S. D. M. 1996, MNRAS, 282, 347

Morales, M. F. 2005, ApJ, 619, 678

Morales, M. F., \& Hewitt, J. 2004, ApJ, 615, 7

Oh, S. P., \& Haiman, Z. 2003, MNRAS, 346, 456

Peebles, P. J. E. 1993, Principles of Physical Cosmology (Princeton: Princeton Univ. Press)

Press, W. H., \& Schechter, P. 1974, ApJ, 187, 425

Ricotti, M., Gnedin, N. Y., \& Shull, J. M. 2002, ApJ, 575, 49

Santos, M. G., Cooray, A., \& Knox, L. 2004, preprint (astro-ph/0408515)

Scalo, J. 1998, in ASP Conf. Ser. 142, The Stellar Initial Mass Function, ed.

G. Gilmore \& D. Howell (San Francisco: ASP), 201

Scott, D., \& Rees, M. J. 1990, MNRAS, 247, 510

Seljak, U., et al. 2005, Phys. Rev. D, 71, 3511

Shapiro, P. R., Giroux, M. L., \& Babul, A. 1994, ApJ, 427, 25

Sheth, R. K., \& Tormen, G. 1999, MNRAS, 308, 119

Sobolev, V. V. 1960, Moving Envelopes of Stars (Cambridge: Harvard Univ. Press)

Spergel, D. N., et al. 2003, ApJS, 148, 175

Tozzi, P., Madau, P., Meiksin, A., \& Rees, M. J. 2000, ApJ, 528, 597

Wouthuysen, S. A. 1952, AJ, 57, 31

Wyithe, J. S. B., \& Loeb, A. 2004a, ApJ, 610, 117 2004b, ApJ, submitted (astro-ph/0407162)

Zaldarriaga, M., Furlanetto, S. R., \& Hernquist, L. 2004, ApJ, 608, 622

Zuo, L. 1992, MNRAS, 258, 45

Zygelman, B. 2005, ApJ, 622, 1356 